\title{
Resonant-frequency tuning of angular vertical comb-driven microscanner
}

\author{
Youngkee Eun ${ }^{1}$, Jongbaeg $\mathrm{Kim}^{1 *}$ and Liwei Lin $^{2}$
}

\begin{abstract}
The resonant-frequency tuning of a self-aligned angular vertical comb-driven electrostatic microscanner is demonstrated by the electromechanical spring effect. The microscanner is fabricated on a silicon-on-insulator wafer using the plastic deformation of silicon. A tuning electr ode is fabricated to be electrically separated from the actuation electrode to tune the resonant frequency by adjusting the applied direct-current voltage bias. The experimentally obtained maximum resonant-frequency shift was 3.2\% when the resonant frequency of $3167 \mathrm{~Hz}$ is reduced to $3066 \mathrm{~Hz}$ when a tuning voltage of $30 \mathrm{~V}$ was applied while maintaining the actuation voltage. The method enables facile frequency tuning without any permanent geometrical modification to the microscanner.
\end{abstract}

Keywords: Frequency tuning; Micromirror; Microscanner; Electrostatic; Silicon-on-insulator

\section{Introduction}

Electrostatic actuators are widely utilized in microelectromechanical systems owing to their various advantages compared to other actuation schemes. Fast response, low power consumption, and large displacement make them suitable for applications such as microscanners and resonators. Furthermore, electrostatic vertical comb-driven actuators are widely adopted because of their large scanning range and high-frequency operation, especially for microscanners. However, it is very difficult to achieve the exact desired resonant frequency for microscanners or oscillators owing to the inevitable dimensional errors existing in the microfabrication processes. Obtaining the exact designed structural dimensions of the microdevice and maintaining the uniformity of the fabricated products are challenging issues. The deviation in the mass or geometrical errors during the microfabrication processes changes the electromechanical characteristics of the microscanner; even small microscale mass deviations or a geometrical error can drastically change the characteristics of the device. Other than the fabrication errors, mechanical fatigue of the torsional springs or changes of operation environments such as temperature and pressure could also vary the resonant frequency of the microscanner

\footnotetext{
* Correspondence: kimjb@yonsei.ac.kr

${ }^{1}$ School of Mechanical Engineering, Yonsei University, 50 Yonsei-ro,

Seodaemun-gu, Seoul 120-749, Republic of Korea

Full list of author information is available at the end of the article
}

during operations. Therefore, feedback control could be adopted to obtain a stable scanning angle during the operation of a microscanner. For the feedback control, an angular displacement sensing element and a resonant frequency tuning element is required.

Because it is very difficult to achieve the exact desired resonant frequency from the fabricated device, a post-fabrication process is generally required to tune the deviated resonant frequency back to the designed value. In order to accomplish this, previous works have increased the movable mass by laser ablation deposition [1], increased the stiffness of the mechanical spring by polysilicon deposition [2], and decreased the stiffness of the in-plane vibrating microstructure by using a focused ion beam [3]. These methods cause permanent modification to the microstructure of the device to adjust the resonant frequency to the desired value.

On the other hand, other studies have been carried out to tune the resonant frequency of the device without permanent structural modifications and therefore offer active and reversible tuning capabilities. Previous efforts to tune the deviated resonant frequency have used the electrostatic spring effect, which is an electrostatic stiffness dependency on the applied voltage $[4,5]$, and the geometry of the capacitors [6-11]. A thermal method that increases the temperature of the device to change the mechanical characteristics of the device to tune the resonant frequency was also introduced $[12,13]$. These frequency-tuning methods 
allow the device to perform its functions at various resonant frequencies. Although electrostatic vertical comb actuators are widely adopted for light scanning applications, only a few frequency-tuning methods such as inducing a compressive stress on the flexures [14] or using an angle limiter near the torsional spring [15] have been reported.

In this paper, a frequency-tuning method for an electrostatic self-aligned angular vertical comb (AVC)-driven microscanner is described. The AVC-based microscanner generates torsional motion of a micromirror with respect to the axis of rotation for optical scanning. A tuning comb electrode that is electrically separated from the driving comb electrode is designed to tune the resonant frequency. The presented frequency-tuning method is based on the electrostatic spring effect induced by the nonlinear relation between the electrostatic moment and the angular displacement of the microscanner. By independently adjusting the direct-current (dc) bias applied to the tuning comb electrode, the tuning capability was maximized under limited voltage source conditions. The microscanner tested for the resonant-frequency-tuning experiments is fabricated via the plastic deformation of single-crystal silicon, as introduced previously [16].

\section{Findings}

A scanning electron microscopy (SEM) image of the AVC-driven microscanner for resonant-frequency tuning is shown in Figure 1. The microscanner is fabricated on a silicon-on-insulator (SOI) wafer by utilizing a plastic deformation process [16] in which the moving comb electrode and fixed comb electrode are vertically interdigitated, forming an initial tilt angle. The rotor is initially tilted in order to generate electrostatic force by introducing an unbalanced electrostatic field in the vertical direction when a voltage difference is applied between the fixed and movable electrodes. The fixed and movable comb finger electrodes are defined on a single photomask and subsequently tilted via plastic deformation of the singlecrystal silicon. Therefore, the comb fingers are inherently self-aligned. The initial tilt angle between the rotor and the stator is $3.1^{\circ}$. The microscanner consists of a rotor, two torsional springs, and two fixed electrodes. The rotor acts as a movable electrode, which is the countering part of the fixed electrodes, and includes a rectangular micromirror with dimensions of $660 \mu \mathrm{m} \times 1200 \mu \mathrm{m}$ for optical scanning. This rotor is supported by the torsional springs that are connected to the anchors. The fixed driving electrode for actuation and the fixed tuning electrode for resonant-frequency tuning are designed to be electrically isolated and symmetrically placed on both sides of the rotor with respect to the torsional springs. The fixed comb finger electrodes are anchored onto the substrate, which is the handle silicon layer of the SOI wafer. The handle

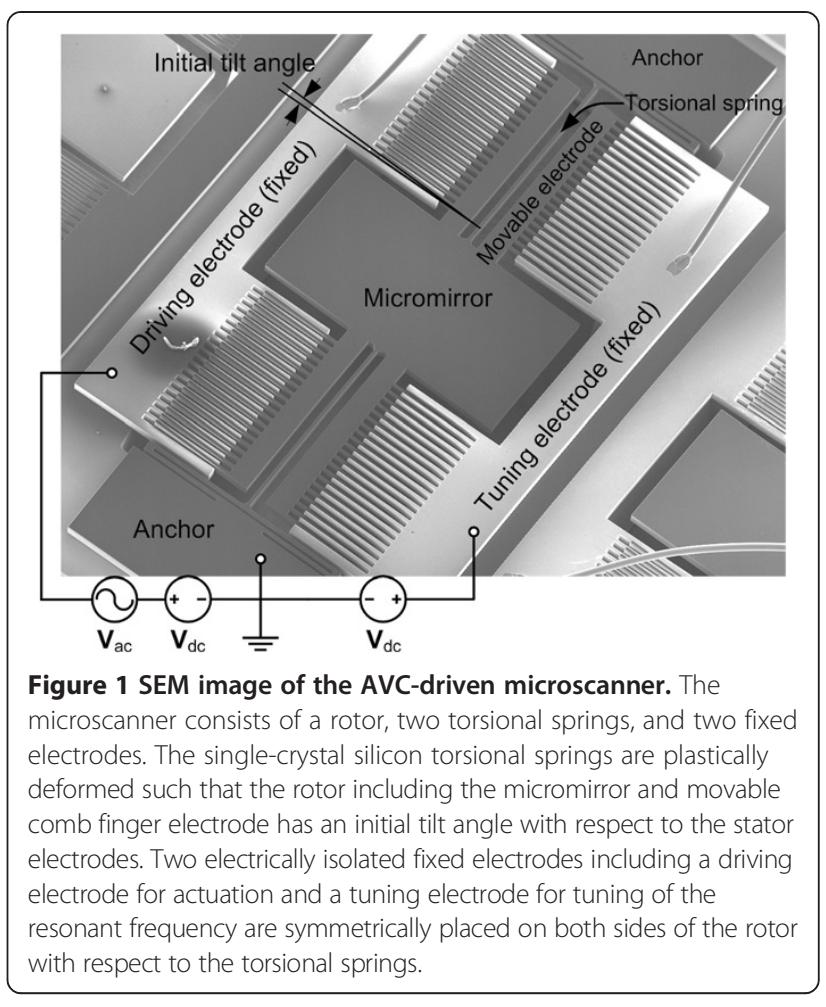

silicon layer below the rotor is completely etched so that the micromirror has enough clearance for its motion. By applying voltage differences between the rotor and the fixed electrodes, the electrostatic moment moves the rotor to find a force-balancing position and therefore induces angular motion of the micromirror.

Figure 2 shows the normalized mechanical and electrostatic moment of the AVC-driven microscanner with respect to the angular displacement. The electrostatic moment (bold black curve) is calculated by a finite element analysis (FEA) using a unit comb electrode-set model to account for the fringing fields. The electrostatic and mechanical moments for the AVC-drive actuator are expressed as

$$
M_{e}=\frac{1}{2} \frac{\partial C}{\partial \theta} V^{2}
$$

$\$ \$\{\mathrm{M}\}_{-} \mathrm{e}=\backslash$ frac $\{1\}\{2\} \backslash$ frac $\{\backslash$ partial $C\}\{\backslash$ partial $\backslash$ theta $\}\{\mathrm{V}\}$ $\wedge 2 \$$

$$
M_{m}=k_{m} \theta
$$

$\$ \$\{\mathrm{M}\} \_\mathrm{m}=\{\mathrm{k}\} \_\mathrm{m} \backslash$ theta $\$ \$$ where $C, \theta, V$, and $k_{m}$ are the capacitance, the angular displacement of the rotor with respect to the stator, the applied voltage, and the mechanical stiffness of the microscanner, respectively. As shown in Figure 2, the electrostatic moment of the AVC-driven actuator is highly dependent on the angular position of the rotor with electrostatic stiffness $k_{e}$. When 


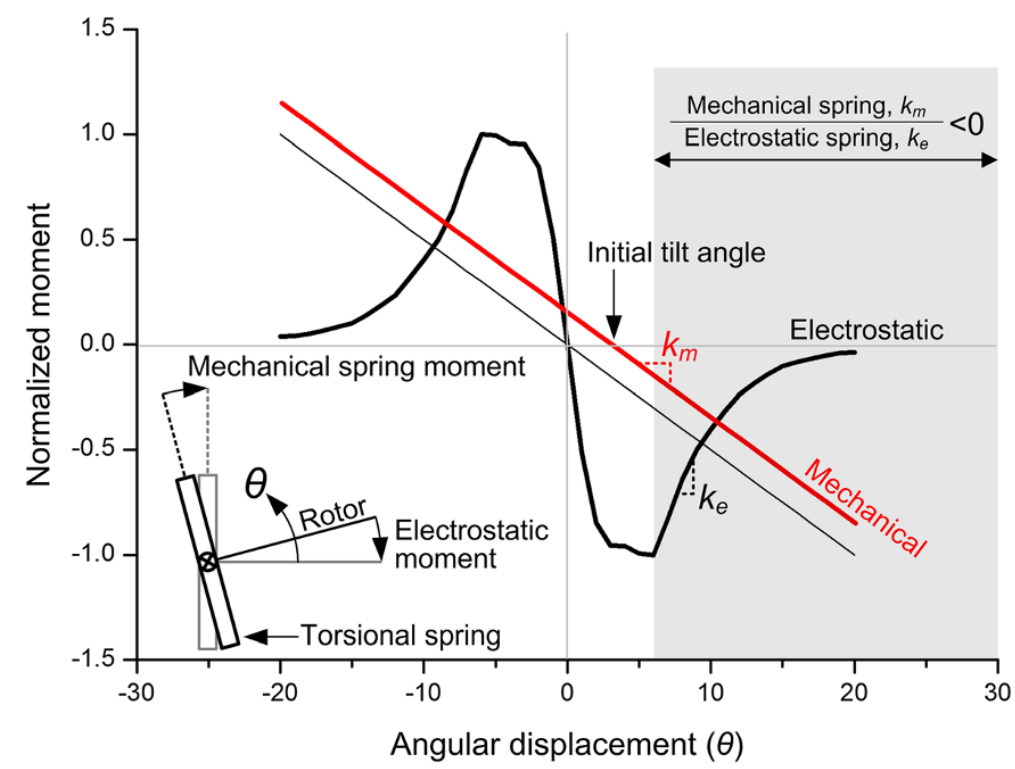

Figure 2 Normalized mechanical and electrostatic moment of the AVC-driven microscanner with respect to the angular displacement. The electrostatic moment (bold black curve) is calculated by FEA, and a unit comb electrode set is used to account for the fringing fields. The mechanical restoring moment of the torsional spring is depicted for the torsional spring with (red line) and without (thin black line) an initial tilt angle.

the angular displacement increases such that the moving and fixed combs begin to disengage, a significant nonlinearity in electrostatic moment occurs with respect to the angular displacement. Furthermore, the electrostatic stiffness also varies with respect to the angular position of the rotor and is proportional to the dc voltage applied to the fixed tuning electrodes. The relationship between the resonant frequency and the stiffness is given as [10]

$$
f=\frac{1}{2 \pi} \sqrt{\frac{k}{J}}=\frac{1}{2 \pi} \sqrt{\frac{k_{m}+k_{e}}{J}}=f_{0} \sqrt{1+\frac{k_{e}}{k_{m}}}
$$

$\$ \$ \quad \mathrm{f}=\backslash \operatorname{frac}\{1\}\{2 \backslash \mathrm{pi}\} \backslash \mathrm{sqrt}\{\mid \operatorname{frac}\{\mathrm{k}\}\{J\}\}=\backslash$ frac $\{1\}\{2 \backslash \mathrm{pi}\} \backslash \mathrm{sqrt}$

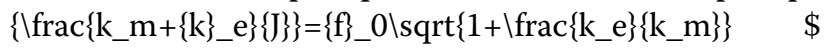
$\$$ where $k, J$, and $f_{o}$ are the combined electromechanical stiffness, the moment of inertia, and the un-tuned resonant frequency of the microscanner, respectively. Considering the angular displacement range of the aforementioned normalized electromechanical moment colored in gray in Figure 2, the value of the electrostatic stiffness is negative, and the value of the mechanical stiffness is positive. As a result, the electromechanical stiffness of the AVC-driven microscanner exhibits a spring-softening behavior and reduces the resonant frequency because the electrostatic and mechanical spring constants are in opposite directions.

As illustrated in Figure 1, the experiment was performed by applying a fixed value of the driving voltage to the fixed driving electrode while varying the value of the tuning $\mathrm{dc}$ voltage applied to the fixed tuning electrode. The rotor of the microscanner was kept electrically grounded. Figure 3(a) shows the frequency response of the microscanner with a fixed actuation voltage consisting of a $10-\mathrm{V}$ alternating-current (ac) driving voltage on top of a $10-\mathrm{V}$ dc bias $\left(\mathrm{V}_{\text {drive }}=10 \mathrm{~V}_{\mathrm{dc}}+10 \mathrm{~V}_{\mathrm{ac}}\right)$ applied to the driving electrodes. The un-tuned resonant frequency was $3152 \mathrm{~Hz}$ when no voltage was applied to the tuning electrodes. The quality factor of the un-tuned microscanner was 112 with a maximum scanning angle of $11^{\circ}$. Subsequently, the tuning voltage was applied, and the tuned resonant frequency was measured. When the tuning voltage of $30 \mathrm{~V}$ was applied for the resonant frequency tuning, the quality factor of the microscanner was 108 with a maximum scanning angle of $6.2^{\circ}$.

In Figure 3(b), the tuned resonant frequency is plotted as a function of the dc tuning voltages over a range of $0 \mathrm{~V}$ to $30 \mathrm{~V}$. It is clearly observed that the magnitude of the frequency shift is proportional to the applied tuning voltages. The maximum resonant-frequency shift achieved with a tuning voltage of $30 \mathrm{~V}$ was $101 \mathrm{~Hz}(3.2 \%)$ from $3167 \mathrm{~Hz}$ with a driving voltage consisting of a $10-\mathrm{V}$ ac bias and a $20-\mathrm{V}$ dc bias $\left(\mathrm{V}_{\text {drive }}=20 \mathrm{~V}_{\mathrm{dc}}+10 \mathrm{~V}_{\mathrm{ac}}\right)$. The resonant frequency shifts as a function of the applied tuning $\mathrm{dc}$ voltages in the range of $0 \mathrm{~V}$ to $30 \mathrm{~V}$. A resonant-frequency shift of $100 \mathrm{~Hz}$ from $3158 \mathrm{~Hz}$ was achieved with a driving voltage of $20 \mathrm{~V}_{\mathrm{dc}}+5 \mathrm{~V}_{\mathrm{ac}}$. In addition, resonant-frequency shifts of $76 \mathrm{~Hz}$ from $3152 \mathrm{~Hz}$ and $75 \mathrm{~Hz}$ from $3149 \mathrm{~Hz}$ were respectively achieved with a driving voltage of 10 $\mathrm{V}_{\mathrm{dc}}+10 \mathrm{~V}_{\mathrm{ac}}$ (black squares) and with a driving voltage of $10 \mathrm{~V}_{\mathrm{dc}}+5 \mathrm{~V}_{\mathrm{ac}}$ for the un-tuned resonant frequency (green 

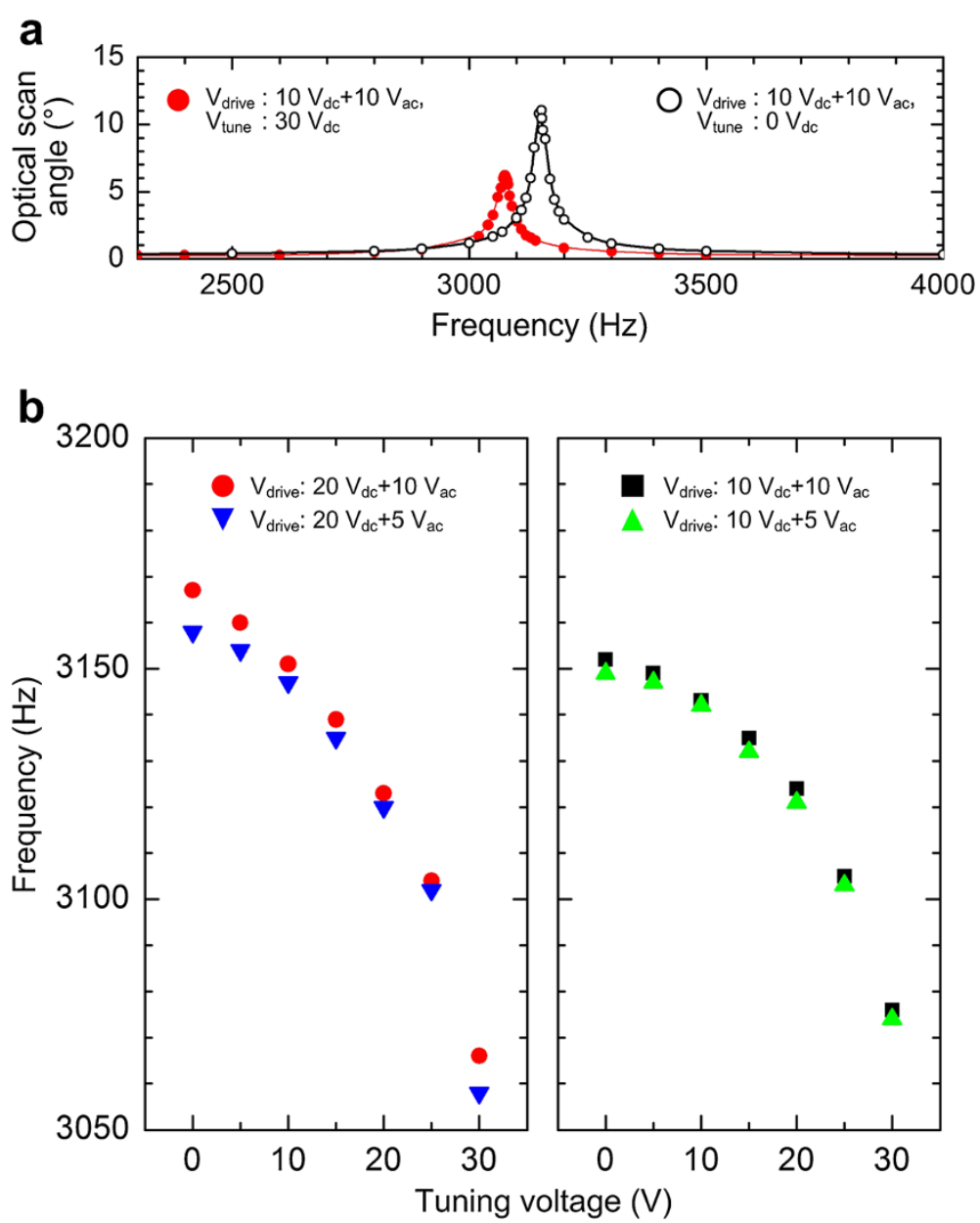

Figure 3 (a) Measured un-tuned and tuned resonant frequencies of the microscanner $(3152 \mathrm{~Hz}$ and $3076 \mathrm{~Hz}$ ) with a driving voltage of $10 \mathrm{~V}_{\mathrm{dc}}+10 \mathrm{~V}_{\mathrm{ac}}$ (b) Measured resonant-frequency shift as a function of the applied tuning dc voltages from $0 \mathrm{~V}$ to $30 \mathrm{~V}$. The resonant frequency is shifted by $101 \mathrm{~Hz}$ from $3167 \mathrm{~Hz}$ for the un-tuned resonant frequency with a driving voltage of $20 \mathrm{~V}_{\mathrm{dc}}+10 \mathrm{Vac}$ (red circles) and by $100 \mathrm{~Hz}$ from $3158 \mathrm{~Hz}$ with a driving voltage of $20 \mathrm{~V}_{\mathrm{dc}}+5 \mathrm{~V}_{\mathrm{ac}}$ (blue inverted triangles). The resonant-frequency shifts of $76 \mathrm{~Hz}$ from $3152 \mathrm{~Hz}$ and $75 \mathrm{~Hz}$ from $3149 \mathrm{~Hz}$ were respectively achieved with a driving voltage of $10 \mathrm{~V}_{\mathrm{dc}}+10 \mathrm{~V}_{\mathrm{ac}}$ (black squares) and with a driving voltage of $10 \mathrm{~V}_{\mathrm{dc}}+5 \mathrm{~V}_{\mathrm{ac}}$ for the un-tuned resonant frequency (green triangles).

triangle). In all cases, the application of the voltage to the tuning electrode reduced the resonant frequency owing to the spring-softening characteristics of the electrostatic spring effect of the given microscanner geometries.

\section{Conclusion}

Frequency tuning of an AVC-driven microscanner was demonstrated by utilizing the electromechanical spring effect. The resonant frequency was tuned by applying a dc tuning voltage up to $30 \mathrm{~V}$ to the tuning comb electrode with four different driving voltages. A maximum frequency shift of $3.2 \%$ was achieved, as the resonant frequency of $3167 \mathrm{~Hz}$ was reduced to $3066 \mathrm{~Hz}$ when a tuning voltage of $30 \mathrm{~V}$ was applied to the tuning comb electrode. Further, the frequency shift of the microscanner was unidirectional. The application of a tuning voltage reduced the resonant frequency because of the spring-softening characteristics of the electromechanical spring effect for the given AVC-driven geometries. By utilizing the electromechanical spring effect of the AVC drive, frequency tuning of the microscanner was achieved without adding any permanent modification to the device structures.

\section{Competing interests}

The authors declare no competing financial interests.

\section{Authors' contributions}

JK conceived the idea and supervised the project. JK and LL discussed the design and the fabrication process of the microscanner. YE performed the frequency-tuning experiments. JK and YE drafted the manuscript. All authors read and approved the final manuscript. 


\section{Acknowledgements}

This research was supported by the Center for Integrated Smart Sensors as Global Frontier Project (CISS-2012M3A6A6054201), the Fusion Research Program for Green Technologies (NRF-2010-0019088) through the National Research Foundation of Korea funded by the Ministry of Science, ICT and Future Planning, and the National Research Foundation of Korea Grant (NRF-2012R1A1A2043661) funded by the Korean Government, and supported (in part) by the Yonsei University Research Fund of 2013.

\section{Author details}

'School of Mechanical Engineering, Yonsei University, 50 Yonsei-ro, Seodaemun-gu, Seoul 120-749, Republic of Korea. ${ }^{2}$ Department of Mechanical Engineering, University of California, Berkeley, CA 94720, USA.

Received: 17 March 2014 Accepted: 12 May 2014

Published online: 17 July 2014

\section{References}

1. Chiao M, Lin $L$ (2004) Post-packaging frequency tuning of microresonators by pulsed laser deposition. J Micromech Microeng 14:1742-1747

2. Joachim D, Lin L (2003) Characterization of selective polysilicon deposition for MEMS resonator tuning. J Microelectromech Syst 12:193-200

3. Syms RRA, Moore DF (1999) Focused ion beam tuning of in-plane vibrating micromechanical resonators. Electron Lett 35:1277-1278

4. Adams SG, Bertsch FM, Shaw KA, MacDonald NC (1998) Independent tuning of linear and nonlinear stiffness coefficients. J Microelectromech Syst 7:172-180

5. Lee WS, Kwon KC, Kim BK, Cho JH, Youn SK (2004) Frequency-shifting analysis of electrostatically tunable micro-mechanical actuator CMES: Comp Model Eng Sci 5:279-286

6. Gallacher BJ, Hedley J, Burdess JS, Harris AJ, Rickard A, King DO (2005) Electrostatic correction of structural imperfections present in a microring gyroscope. J Microelectromech Syst 14:221-234

7. Lee KB, Cho YH (1998) A triangular electrostatic comb array for micromechanical resonant frequency tuning. Sensor Actuat A Phys 70:112-117

8. Jensen BD, Mutlu S, Miller S, Kurabayashi K, Allen JJ (2003) Shaped comb fingers for tailored electromechanical restoring force. J Microelectromech S 12:373-383

9. Lee KB, Lin L, Cho YH (2008) A closed-form approach for frequency tunable comb resonators with curved finger contour. Sensor Actuat A Phys 141:523-529

10. Morgan B, Ghodssi R (2008) Vertically-shaped tunable MEMS resonators. J Microelectromech Syst 17:85-92

11. Scheibner D, Mehner J, Reuter D, Kotarsky U, Gessner T, Dötzel W (2004) Characterization and self-test of electrostatically tunable resonators for frequency selective vibration measurements. Sensor Actuat A Phys 111:93-99

12. Syms RRA (1998) Electrothermal frequency tuning of folded and coupled vibrating micromechanical resonators. J Microelectromech Syst 7:164-171

13. Remtema $T$, Lin $L$ (2001) Active frequency tuning for micro resonators by localized thermal stressing effects. Sensor Actuat A Phys 91:326-332

14. Shmilovich T, Krylov S (2008) Linear tuning of the resonant frequency in tilting oscillators by an axially loaded suspension flexure. In: IEEE 21 st International Conference on Micro Electro Mechanical Systems. Tucson, AZ, pp 657-660

15. Elata D, Leus V, Hirshberg A, Salomon O, Naftali M (2007) A novel tilting micromirror with a triangular waveform resonance response and an adjustable resonance frequency for raster scanning applications. In: International Solid-State Sensors, Actuators and Microsystems Conference. Lyon, pp 1509-1512

16. Kim J, Lin L (2005) Electrostatic scanning micromirrors using localized plastic deformation of silicon. J Micromech Microeng 15:1777-1785

\section{doi:10.1186/s40486-014-0004-9}

Cite this article as: Eun et al:: Resonant-frequency tuning of angular vertical comb-driven microscanner. Micro and Nano Systems Letters $20142: 4$

\section{Submit your manuscript to a SpringerOpen ${ }^{\circ}$ journal and benefit from:}

- Convenient online submission

- Rigorous peer review

- Immediate publication on acceptance

- Open access: articles freely available online

- High visibility within the field

- Retaining the copyright to your article

Submit your next manuscript at $>$ springeropen.com 